\title{
A GENERALISATION OF THE RADON-NIKODYM THEOREM
}

\author{
P. D. FINCH
}

(received 10 January 1964, revised 6 August 1964)

\section{Introduction}

Let $\mathscr{X}$ be a space of points $x, \mathscr{H}$ a $\sigma$-field of subsets of $\mathscr{X}$ and $\mu$ a $\sigma$-finite measure on $\mathscr{K}$. The elements of $\mathscr{H}$ will be called measurable sets and all the sets considered in this paper are measurable sets. A real-valued point function $\mathrm{t}(x)$ on $\mathscr{X}$ will be said to be measurable if, for each real $\alpha$, the set $\{x: \mathrm{t}(x) \leqq \alpha\}$ is measurable. Let $\mathscr{K}(S), S \subset \mathscr{X}$ denote the $\sigma$-field of all measurable subsets of $S$. A real-valued function $f(\cdot)$ on $\mathscr{K}$ will be called a set function.

In Finch [1] a theory of integration of set functions $f(M), M \in \mathscr{K}$ with respect to the measure $\mu$ is developed. In that theory the integral

$$
I_{f}(S)=(I) \int_{\mathcal{M}(S)} f(M) \mu(M)
$$

is, when it exists, the limit of the approximating sums

$$
F_{\Pi}(S)=\sum_{\Pi(S)} f(M) \mu(M)
$$

where the summation is over all elements with positive $\mu$-measure, of the partition $\Pi(S)$ of $S$ by elements of $\mathscr{M}(S)$ and the limit is taken in the sense of Moore-Smith convergence as the partitions spread. For details of the theory we refer to Finch [1] where it is shown that the $\Pi$-integral (1.1) is, when it exists, a $\sigma$-additive set function on $\mathscr{K}$, that is,

$$
I_{f}\left\{\sum_{j=1}^{\infty} M_{j}\right\}=\sum_{j=1}^{\infty} I_{f}\left(M_{j}\right)
$$

whenever the sets $M_{j}$ are mutually disjoint elements of $\mathscr{M}$. Thus $I_{f}$ is a signed measure on $\mathscr{K}$ and it follows from (1.2) that it is absolutely continuous with respect to $\mu$. It follows from the Radon-Nikodym theorem that there is a measurable point function $i_{f}(x)$ on which is finite except possibly on a set of $\mu$-measure zero, such that

$$
I_{f}(S)=(L) \int_{S} i_{f}(x) d \mu(x)
$$


where the $L$-integral is the Lebesgue integral of $i_{f}(x)$ with respect to the measure $\mu$. Further if $j_{f}(x)$ is any other measurable point function satisfying (1.4) then

$$
\mu\left\{x: j_{f}(x) \neq i_{f}(x)\right\}=0 .
$$

It is of interest therefore to examine the relationship between the $\Pi$ integrable set function $f$ and the associated point function $i_{f}$. A partial solution to this problem is provided by the following theorem to whose proof this paper is devoted.

THEOREM 1. Let $\mathscr{X}$ be a space of points $x, \mathscr{H}$ a $\sigma$-field of subsets of $\mathscr{X}$ and $\mu$ a $\sigma$-finite measure on $\mathscr{M}$. Let $\nu$ be a $\sigma$-finite signed measure on $\mathscr{M}$ and let $g(\xi)$ be a real-valued function of bounded variation of the variable $\xi$. Write

$$
f(M)=g\{v(M) / \mu(M)\}, \quad M \in \mathscr{M}, \quad \mu(M)>0,
$$

then there exists a real-valued measurable point function $\theta(x)$ on $\mathscr{X}$ which is finite, except possibly on a set of $\mu$-measure zero, such that for each $S$

$$
(I) \int_{\mathcal{M}(S)} f(M) \mu(M)=(L) \int_{S} g\{\theta(x)\} d \mu(x)
$$

whenever either integral exists.

Remarks. Since $\mathscr{X}$ is the countable union of disjoint elements of $\mathscr{M}$ on which $\mu$ and $\nu$ are each finite it is sufficient to prove the theorem when $\mu$ and $\nu$ are each finite. Secondly it is clearly sufficient to prove the theorem when the function $g$ is monotonic and non-negative. From here on, therefore, we shall assume that $\mu$ is a finite measure, $v$ is a finite signed measure and that $g$ is monotonic non-decreasing and non-negative.

Note that the theorem does not assert that the function $g\{\theta(x)\}$ is $L$-integrable with respect to $\mu$, in fact a necessary and sufficient condition for this is the existence of the $I I$-itegral in (1.6). Note also that the statement of the theorem does not assert that the signed measure $v$ is absolutely continuous with respect to $\mu$. However the $L$-integrability of $\theta(x)$ or equivalently the existence of the $\Pi$-integral $(1.6)$ when $g(\xi) \equiv \xi$ is a necessary and sufficient condition for the absolute continuity of $v$ with respect to $\mu$.

To see this observe that when $g(\xi) \equiv \xi$ the approximating sum (1.2) to the $I$-integral (1.6) is

$$
F_{\Pi}(S)=\sum_{\Pi(S)} v(M)
$$

where the summation is over those elements $M$ of the partition $\Pi(S)$ with $\mu(M)>0$. If $v$ is absolutely continuous with respect to $\mu$ then $F_{n}(S)=\nu(S)$ since $\mu(M)=0$ implies $\nu(M)=0$ and the $\Pi$ integral exists and has the value $v(S)$.

Conversely if the $\Pi$-integral exists, that is, if the $\Pi$-limit of (1.7) exists 
this limit is unique. Choosing a sequence of partitions $\left\{\Pi_{n}(S)\right\}$ of $S$ with $\Pi_{n+1}(S)$ finer than $\Pi_{n}(S)$ and such that each element of the partition $\Pi_{n}(S)$ has positive $\mu$-measure we see that this limit is $\nu(S)$. Let $S_{0}$ be any element of $\mathscr{M}$ with $\mu\left(S_{0}\right)=0$ and write $S_{1}=S_{U} S_{0}$. Choosing a sequence of partitions $\left\{\Pi_{n}\left(S_{1}\right)\right\}$, of $S_{1}$ such that each element of $\Pi_{n}\left(S_{1}\right)$ has positive $\mu$-measure and $\Pi_{n+1}\left(S_{1}\right)$ is finer that $\Pi_{n}\left(S_{1}\right)$ we obtain the limit $v\left(S_{1}\right)$. Since the $\Pi$-integral has a unique value $\nu\left(S_{1}\right)=\nu(S)$, that is, $\nu\left(S_{0}\right)=0$ and this shows that $\nu$ is absolutely continous with respect to $\mu$.

It follows from the above that theorem 1 , contains the Radon-Nikodym theorem as a particular case and for this reason our proof of it does not depend on the Radon-Nikodym theorem. An example showing that the theorem can be true when $\nu$ is not absolutely continuous with respect to $\mu$, in fact when $\mu$ is absolutely continuous with respect to $\nu$ is given in section 3 . One use of theorem 1 is that it reduces the calculation of the $\Pi$-integral to that of an $L$-integral, such a use is illustrated in section 4 by application to a problem in information theory.

\section{Some preliminary results}

In this section we state some preliminary results which are required for the proof of theorem 1 .

Lemma (2.1). Let $R$ denote the set of real numbers and let $\left\{\alpha_{j}\right\}$ be a sequence of real numbers which is dense in $R$. Suppose that $\left\{M\left(\alpha_{j}\right)\right\}$ is a family of elements of $\mathscr{M}$, indexed by the dense sequence $\alpha_{j}$ and such that

(i) $M\left(\alpha_{i}\right) \subset M\left(\alpha_{j}\right)$ if $\alpha_{i}<\alpha_{j}$

(ii) $M\left(\alpha_{i}\right)=\bigcap_{\alpha_{j}>\alpha_{i}} M\left(\alpha_{j}\right)$

For any real $\alpha$ define

$$
M(\alpha)=\bigcap_{\alpha_{j}>\alpha} M\left(\alpha_{j}\right)
$$

then there exists a real-valued measurable point function $\theta(x)$ on $\mathscr{X}$ such that

$$
M(\alpha)=\{x: \theta(x) \leqq \alpha\} .
$$

If further

(iii) $\lim _{\alpha \rightarrow \infty} \mu\{\mathscr{X}-M(\alpha)\}=0, \lim _{\alpha \rightarrow \infty} \mu\{M(-\alpha)\}=0$,

then $\theta(x)$ is finite except possibly on a set of $\mu$-measure zero.

This lemma is proved easily by writing

$$
\theta(x)=\inf \{\alpha: x \in M(\alpha)\}
$$

Using lemma (2.1) one may prove

Lemma (2.2). If $\mu$ is a finite measure on $\mathscr{M}, v$ is a finite signed measure on $\mathscr{M}$ then there exists a measurable point function $\theta(x)$ on $\mathscr{M}$ which is finite 
except possibly on a set of $\mu$-measure zero, such that if for each real $\alpha$,

$$
M(\alpha)=\{x: \theta(x) \leqq \alpha\}
$$

then

$$
\begin{array}{ll}
\nu(M) \leqq \alpha \mu(M), & M \subset M(\alpha) \\
\nu(M) \geqq \alpha \mu(M), & M \subset \mathscr{X}-M(\alpha) .
\end{array}
$$

Proof. For each real $\alpha$ and each $M \in \mathscr{K}$ write

$$
\lambda(M ; \alpha)=\nu(M)-\alpha \mu(M)
$$

Let $\left\{\alpha_{j}\right\}$ be a dense sequence of real numbers, for each $\alpha_{j}, \lambda\left(M ; \alpha_{j}\right)$ is a finite signed measure on $\mathscr{H}$ and so, by the Hahn decomposition of $\mathscr{X}$ with respect to this signed measure, there exists an element $M\left(\alpha_{j}\right)$ of $\mathscr{M}$ such that

$$
\begin{array}{ll}
\lambda\left(M ; \alpha_{j}\right) \leqq 0, & M \subset M\left(\alpha_{j}\right) \\
\lambda\left(M ; \alpha_{j}\right) \geqq 0, & M \subset \mathscr{X}-M\left(\alpha_{j}\right) .
\end{array}
$$

The proof of lemma (2.2) consists in verifying that we can choose the sets $M\left(\alpha_{j}\right)$ to satisfy the conditions of lemma $(2.1)$. Since this verification uses standard procedures, for example, Royden [3], it will be omitted.

\section{Proof of theorem 1}

We proceed now to the proof of theorem 1 . Since $g$ is non-negative and monotonic non-decreasing the inequality

$$
f(M) \leqq \alpha, \quad M \in \mathscr{K}, \quad \mu(M)>0
$$

is equivalent to the inequality

$$
\nu(M)-\left(g^{-1} \alpha\right) \mu(M) \leqq 0, \quad M \in \mathscr{M}, \quad \mu(M)>0 .
$$

Here and in what follows

$$
g^{-1} \alpha=\sup \{\xi: g(\xi) \leqq \alpha\} .
$$

Thus if $\theta(x)$ is the measurable point function of lemma (2.2) we have,

$$
\begin{array}{lll}
f(M) \leqq \alpha & \text { if } & M \subset M\left(g^{-1} \alpha\right) \\
f(M) \geqq \alpha & \text { if } & M \subset \mathscr{X}-M\left(g^{-1} \alpha\right)
\end{array}
$$

where

and

$$
M\left(g^{-1} \alpha\right)=\left\{x: \theta(x) \leqq g^{-1} \alpha\right\}
$$

$$
g\{\theta(x)\}=\inf \left\{\alpha: x \in M\left(g^{-1} \alpha\right)\right\} .
$$

Let $\delta$ be an arbitrary positive real number and let $\left\{\delta_{j}\right\}, j=0,1, \cdots$ be a sequence of real numbers with $\delta_{0}=0$, and such that 


$$
0<\delta_{j}-\delta_{j-1} \leqq \delta, \quad j \geqq 1, \quad \text { and } \sup \delta_{j}=+\infty
$$

Write

$$
M_{j}=\left\{x: \delta_{j-1}<g\{\theta(x)\} \leqq \delta_{j}\right\},
$$

Then for any $j$ such that $\mu\left(M_{j}\right)>0$ we have

$$
\delta_{j-1} \leqq f(M) \leqq \delta_{j}, \quad M \in \mathscr{M}, \quad M \subset M_{j}, \quad \mu(M)>0 .
$$

It follows that the total variation

$$
|f|(M)=\sup \left\{f\left(A_{1}\right)-f\left(A_{2}\right): \mu\left(A_{i}\right)>0, A_{i} \subset M\right\}
$$

on $f$ on $\mathscr{K}$ with respect to $\mu$ does not exceed $\delta$ on the measurable subsets of each $M_{j}$. Thus

$$
|f|(M) \leqq \delta, \quad M \in \mathscr{M}, M \subset M_{j} ; \quad \mu(M)>0 .
$$

Write

$$
f^{(n)}(M)=\left\{\begin{array}{lll}
f(M) & \text { if } & f(M) \leqq n \\
n & \text { if } & f(M) \geqq n
\end{array}\right.
$$

for each $M \in \mathscr{H}$ with $\mu(M)>0$.

Let $S$ be any element of $\mathscr{M}$, then

$$
\Pi(S)=\left\{S M_{j}\right\}, \quad j=0,1,2, \cdots
$$

is a partition of $S$. It follows from (3.4) and theorem (3.3) of Finch [1], that $f^{(n)}(\cdot)$ is $\Pi$-integrable on $\mathscr{M}(S)$ with respect to $\mu$, that is,

$$
\begin{aligned}
(I I) & \int_{\mathscr{M}(S)} f^{(n)}(M) \mu(M) \\
& =(\Pi) \lim \sum_{j=-\infty}^{+\infty} f^{(n)}\left(S M_{j}\right) \mu\left(S M_{j}\right)
\end{aligned}
$$

exists.

Because of (3.2) and (3.3) it follows also that the sum on the right-hand side of (3.5) is an approximating sum for the Lebesgue integral

$$
\text { (L) } \int_{S} g^{(n)}\{\theta(x)\} d \mu(x)
$$

where

$$
g^{(n)}(\xi)=\left\{\begin{array}{lll}
g(\xi) & \text { if } & g(\xi) \leqq n \\
n & \text { if } & g(\xi)>n
\end{array}\right.
$$

and hence that

$$
(I) \int_{\mu(S)} f^{(n)}(M) \mu(M)=(L) \int_{S} g^{(n)}\{\theta(x)\} d \mu(x)
$$


Letting $n \rightarrow \infty$ in (3.6) we obtain (1.6) whenever either integral exists. The uniqueness of $\theta(x)$ follows immediately since if $\varphi(x)$ is another such measurable point function

$$
\text { (L) } \int_{S}\left[g^{(n)}\{\theta(x)\}-g^{(n)}\{\varphi(x)\}\right] d \mu(x)=0
$$

for all $S \in \mathscr{M}$ and each $n>0$, hence

$$
\mu\{x ; \phi(x) \neq \theta(x)\}=0 .
$$

This completes the proof of the theorem.

As remarked in section one the formulation of theorem 1 does not introduce explicitly the condition that $\nu$ should be absolutely continuous with respect to $\mu$, although, as we have shown, if the $\Pi$-integral (1.6) exists when $g(\xi) \equiv \xi$ this implies that the signed measure $v$ is absolutely continuous with respect to $\mu$. To illustrate that meaningful results may be obtained when $\nu$ is not absolutely continuous with respect to suppose in fact that $\nu$ and $\mu$ are both finite measures and that $\mu$ is absolutely continuous with respect to $\nu$ with density $\phi(x)$, so that

$$
\mu(M)=(L) \int_{M} \phi(x) d v(x) .
$$

Suppose also that $\phi(x)$ belongs to the class $L_{p}(v)$ for some $p>1$, so that

$$
(L) \int_{M}\{\phi(x)\}^{p} d v(x)
$$

exists for each $M \in \mathscr{M}$.

Consider the identity

$$
\{\mu(M) / v(M)\}^{p} v(M)=\{v(M) / \mu(M)\}^{-(p-1)} \mu(M)
$$

where $\nu(M)>0$. By applying theorem 1 to the left-hand side we obtain

$$
(\Pi) \int_{\mathcal{M}(S)}\{\mu(M) / \nu(M)\}^{p} \nu(M)=(L) \int_{S}\{\phi(x)\}^{p} d v(x)
$$

Since $\mu$ is absolutely continuous with respect to $v$

$$
\sum_{\nu(M)>0}\left\{\frac{\mu(M)}{\nu(M)}\right\}^{p} \nu(M)=\sum_{\mu(M)>0}\left\{\frac{\nu(M)}{\mu(M)}\right\}^{-p-1} \mu(M)
$$

where the summations are over the elements of the partition $I I(S)$ of $S \in \mathscr{M}$ with positive $v$ and $\mu$ measure respectively. Thus the $\Pi$-integral of which the right-hand side is the approximating sum exists and equals the $\Pi$-integral of (3.7), that is,

$$
(\Pi) \int_{\mathscr{M}) S}\{\nu(M) / \mu(M)\}^{-(p-1)} \mu(M)=(\Pi) \int_{\mathcal{M}_{(S)}}\{\mu(M) / \nu(M)\}^{p_{\nu}}(M) .
$$


Since the $\Pi$-integral on the left-hand side of (3.8) exists theorem 1 ensures the existence of $\theta(x)$ such that

$$
\begin{aligned}
(I) \int_{\mathscr{M}(S)}\{\nu(M) / \mu(M)\}^{-(p-1)} \mu(M) & \\
& =(L) \int_{S}\{\theta(x)\}^{-(p-1)} d \mu(x) .
\end{aligned}
$$

In fact it is clear that $\theta(x)=\{\phi(x)\}^{-1}$ except on a set of $y$ measure zero. Equation (3.9) is the desired example of theorem 1 when $v$ is not absolutely continuous with respect to $\mu$.

\section{An application to information theory}

Let $\mathscr{X}$ be a space of points $x, \mathscr{M}$ a $\sigma$-field of subsets of $\mathscr{X}$ and let $\left\{P\left(\cdot \mid \theta_{j}\right)\right\}, j=1,2, \cdots, k$, be a finite family of probability measures on $\mathscr{M}$. We write $\theta^{(k)}=\left(\theta_{1}, \theta_{2}, \cdots, \theta_{k}\right)$, call the $\theta_{j}$ indices or index values and refer to $\theta^{(k)}$ as the indexing set. The elements of $\mathscr{H}$ we refer to as events. For each $\theta \in \theta^{(k)}$ we call the ordered pair $\{\mathscr{X}, P(\cdot \theta \theta)\}$ a probability space.

In Finch [2] it is shown that an appropriate measure of the amount of conditional information about the particular probability space $\left\{\mathscr{X}, P\left(\cdot \mid \theta_{j}\right)\right\}$ provided by the occurrence of the event $M$ when it is known that $\theta \in \theta^{(k)}$ is given by

$$
\begin{aligned}
& I\left[\{X, P(\cdot \mid \theta)\}: M \mid \theta \in \theta^{(k)}\right] \\
& \quad=-\log \left[P(M \mid \theta) / \sum_{j=1}^{k} P\left(M \mid \theta_{j}\right)\right], \quad \theta \in \theta^{(k)}
\end{aligned}
$$

The quantity

$$
G\left(M \mid \theta^{(k)}\right)=k^{-1} \sum_{j=1}^{k} P\left(M \mid \theta_{j}\right),
$$

is a probability measure over $\mathscr{M}$ and, according to Finch [2], can be interpreted as the generalised probability that the event $M$ occurs under the logical disjunction of hypotheses $\theta_{1} \vee \theta_{2} \vee \cdots \vee \theta_{k}$.

The quantity (4.1) defines an amount of information provided by the occurrence of a particular event $M \in \mathscr{M}$. In order to define an average amount of information it is natural to introduce the quantity

$$
\begin{aligned}
E \cdot I\left[\{\mathscr{X}, P(\cdot \mid \theta)\} \mid \theta^{(k)}\right] & \\
& =(\Pi) \int_{\mathscr{M}} I\left[\{\mathscr{X}, P(\cdot \mid \theta)\}: M \mid \theta \in \theta^{(k)}\right] G\left(M \mid \theta^{(k)}\right),
\end{aligned}
$$

for each $\theta \in \theta^{(k)}$ whenever the $\Pi$-integral exists. The quantity (4.3) is the expected amount of conditional information about the probability space $\{\mathscr{X}, P(\cdot \mid \theta)\}$ provided by an experiment, whose possible outcomes are the events of $\mathscr{M}$, when it is known that $\theta \in \theta^{(k)}$.

The quantity

$$
a\left(\theta \mid M ; \theta^{(k)}\right)=P(M \mid \theta) / \sum_{j=1}^{k} P\left(M \mid \theta_{j}\right), \quad \theta \in \theta^{(k)}
$$


is called the acceptability of the index value $\theta$ in the light of the occurrence of the event $M$ when it is known that $\theta \in \theta^{(k)}$. In terms of the acceptabilities we may rewrite equation (4.3) in the form

$$
\begin{aligned}
E \cdot I\left[\{\mathscr{X}, P(\cdot \mid \theta)\} \mid \theta^{(k)}\right] & \\
& =-(\Pi) \int_{\mathscr{M}} \log \left\{a\left(\theta \mid M ; \theta^{(k)}\right\} G\left(M \mid \theta^{(k)}\right) .\right.
\end{aligned}
$$

It follows from theorem 1 that when this $\Pi$-integral exists there is a realvalued measurable point function on $\mathscr{X}$, a $\left(\theta \mid x ; \theta^{(k)}\right)$ which is finite, except possibly on a set of $G\left(\cdot \mid \theta^{(k)}\right)$ measure zero, such that

$$
\begin{aligned}
& E \cdot I\left[\{X, P(\cdot \mid \theta)\} \mid \theta \in \theta^{(k)}\right] \\
& \quad=-(L) \int_{\mathscr{X}} \log \left\{a\left(\theta \mid x ; \theta^{(k)}\right)\right\} \cdot G\left(d x \mid \theta^{(k)}\right)
\end{aligned}
$$

and where the Lebesgue integral exists if and only if the $\Pi$-integral (4.5) exists.

Since the probability measure $P(\cdot \mid \theta)$ is absolutely continuous with respect to the probability measure $G\left(\cdot \mid \theta^{(k)}\right)$ for each $\theta \in \theta^{(k)}$ it follows from the proof of theorem 1 also, that the point function $a\left(\theta \mid x ; \theta^{(k)}\right)$ is in fact the density of $P(\cdot \mid \theta)$ with respect to the measure $k G\left(\cdot \mid \theta^{(k)}\right)$. Thus theorem 1 reduces the calculation of the $\Pi$-integral (4.5) to that of the $L$-integral (4.6).

\section{References}

[1] Finch, P. D., Integration of real-valued set functions in abstract spaces, This Journal 4 (1964), 202-213.

[2] Finch P. D., The theory of information and statistical inference I, Journ. App. Prob. I (1964), 121-140.

[3] Royden H. L., Real Analysis. Macmillan, New York (1963).

Monash University 